\title{
The Dust Distribution Immediately Surrounding V Hydrae
}

\author{
C. H. Townes, E. H. Wishnow, AND V. Ravi \\ Space Sciences Laboratory and Department of Physics, University of California, Berkeley, CA 94720; \\ cht@ssl.berkeley.edu,wishnow@ssl.berkeley.edu \\ Received 2011 March 24; accepted 2011 October 24; published 2011 November 11
}

\begin{abstract}
Dust surrounding the star V Hydrae has been measured at a $11.15 \mu \mathrm{m}$ wavelength using the threetelescope Infrared Spatial Interferometer (ISI). The narrowband heterodyne detection system is tuned to a region free of spectral lines due to molecules surrounding the star, and only continuum radiation from the dust and star is measured. Closure-phase data show that the dust is symmetrically distributed around the star. Results obtained in 2006-2007 are well fitted by a model with a Gaussian intensity distribution with HWHM of 49 mas that contributes 0.52 of the total flux and by a uniform disk of radius 287 mas with a total flux fraction of 0.26 ; the remaining 0.22 of the total flux is due to the star, which is unresolved. Visibility measurements conducted in 1997 show a substantial difference from those of 2006-2007, indicating that the star was surrounded by more dust in 1997 .
\end{abstract}

\section{INTRODUCTION}

V Hya is a carbon-rich Mira star that has been known for some time to eject substantial material at relatively high velocity. This star is notable for having two variability periods of about 530 and 6000 days (Knapp et al. 1999). Previous size and morphology measurements have been made at near-IR (Millan-Gabet et al. 2003; Ragland et al. 2006), visible and millimeter (Sahai et al. 2003), mid-IR (Lagadec et al. 2005), and submillimeter wavelengths (Hirano et al. 2004), giving information on the stellar size and on the surrounding material. Spectroscopic measurements of very high velocity outflows (up to $240 \mathrm{~km} \mathrm{~s}^{-1}$ ) have been reported by Sahai et al. (2003) and Hirano et al. (2004) and also by Sahai et al. (2009) in the wavelength region of the $\mathrm{CO}$ fundamental band.

We report here on mid-IR interferometric measurements, providing information on the distribution of dust surrounding the star and changes in this surrounding material. Measurements were made using the three-telescope Infrared Spatial Interferometer (ISI) located on Mount Wilson, with baseline separations ranging from 2 to $35 \mathrm{~m}$. The ISI uses heterodyne detection at a wavelength of $11.15 \mu \mathrm{m}$ (Hale et al. 2000). The heterodyne detection, being very narrowband and tunable, allows avoidance of strong molecular spectral lines and, hence, detection of only the dust and stellar continuum radiation.

Previous observations by Sahai et al. (2003) using the Hubble Space Telescope long-slit spectroscopy showed a very high velocity jet, $\sim-240 \mathrm{~km} \mathrm{~s}^{-1}$, observed in [S II] emission to the east of the central continuum source at an angular distance of $\sim 0.2^{\prime \prime}$ (Sahai et al. 2003). In addition, they obtained a CO $J=1-0$ emission map that is elongated in the north-south direction by about $10^{\prime \prime}$. They model these observations with a disk or torus, with its axis in the east-west direction, and with the jet emitted along the axis. Additional $\mathrm{CO}$ emission studies, also with a few-arcsecond angular resolution, show an elongated image in the north-south direction, high bipolar velocities of over $150 \mathrm{~km} \mathrm{~s}^{-1}$ along the east-west axis, and an intermediatevelocity gas of 40-120 $\mathrm{km} \mathrm{s}^{-1}$ (Knapp et al. 1997; Hirano et al. 2004). Broadly speaking, these observations are consistent with a tilted dust disk surrounding the star, which collimates a jet. The origin of the intermediate-velocity wind is not understood, and it may be due to interactions between the high velocity jet and the normal asymptotic giant branch expanding winds, or another outflow, perhaps with a larger cone angle (Hirano et al. 2004). Spectral studies of the CO fundamental band show a range of high velocities, consistent with collimated winds from the source, but they do not indicate different emission cone angles (Sahai et al. 2009). Mid-IR images probing regions closer to the star than the CO images show a slight elongation in the eastwest direction at flux levels below $5 \%$ of the peak surface brightness, but over the central arcsecond they are rather symmetric (Lagadec et al. 2005). Near-IR interferometry measurements of asymmetry using closure phase were reported as being inconsistent (Ragland et al. 2006). Near-IR interferometry combined with Keck aperture masking did not show an elongation, and these measurements were modeled with a uniform stellar disk of 14.5 mas diameter surrounded by a Gaussian dust shell with a FWHM of 35 mas (Millan-Gabet et al. 2003).

\section{MEASUREMENTS AND MODEL FITTING}

A record of the ISI observations is given in Table 1. Observations performed in early 2006 used a linear east-west array with 4,8 , and $12 \mathrm{~m}$ telescope baselines, allowing measurement of structure on spatial scales between 0.05-0.7". Measurements in late 2006 and early 2007 were made using a triangular array configuration with $\sim 35 \mathrm{~m}$ baselines, thereby covering spatial scales as small as about $0.02^{\prime \prime}$. With three baselines, phase 
TABLE 1

TABLE OF OBSERVATIONS

\begin{tabular}{|c|c|c|}
\hline Date & Baseline & Luminosity phase \\
\hline 1997 Oct 27 & $16 \mathrm{~m} \mathrm{E}-\mathrm{W}$ & 0.4 \\
\hline 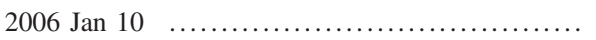 & $4,8,12 \mathrm{~m} \mathrm{E}-\mathrm{W}$ & 0.9 \\
\hline 2006 Nov 20, Dec 21; 2007 Jan 4, 9, 17, $23 \ldots \ldots$ & $35 \mathrm{~m}$ triangle & 0.6 \\
\hline
\end{tabular}

closure was obtained, providing a determination of the asymmetry in the dust distribution. The present ISI measurements of dust on subarcsecond scales show no evidence for asymmetry, as the closure phase was $0^{\circ} \pm 2^{\circ}$ for the linear array configuration. The triangular configuration also gave a closure phase consistent with zero, but the measurements are noisy. Hence, dust close to the star, which is observed here, is assumed to have a spherically symmetric distribution.

Figure 1 shows both the visibility data and a theoretical fit for the data of 2006 and 2007. Data from 1997, denoted by circles, is not included in the fit. The model consists of three uniform disks (UDs). The star is fitted with an assumed disk diameter of $0.015^{\prime \prime}$, based on previous near-infrared interferometry measurements (Millan-Gabet et al. 2003; Pedretti et al. 2005), and it contributes 0.27 of the total flux of the star and surrounding material. The larger dust shell is fitted with a uniform disk of radius $0.268^{\prime \prime}$ with 0.30 of the total flux. The smaller dust shell is fitted with a uniform disk of radius $0.075^{\prime \prime}$ and a flux that is 0.43 of the total. The fit results, including the fitted

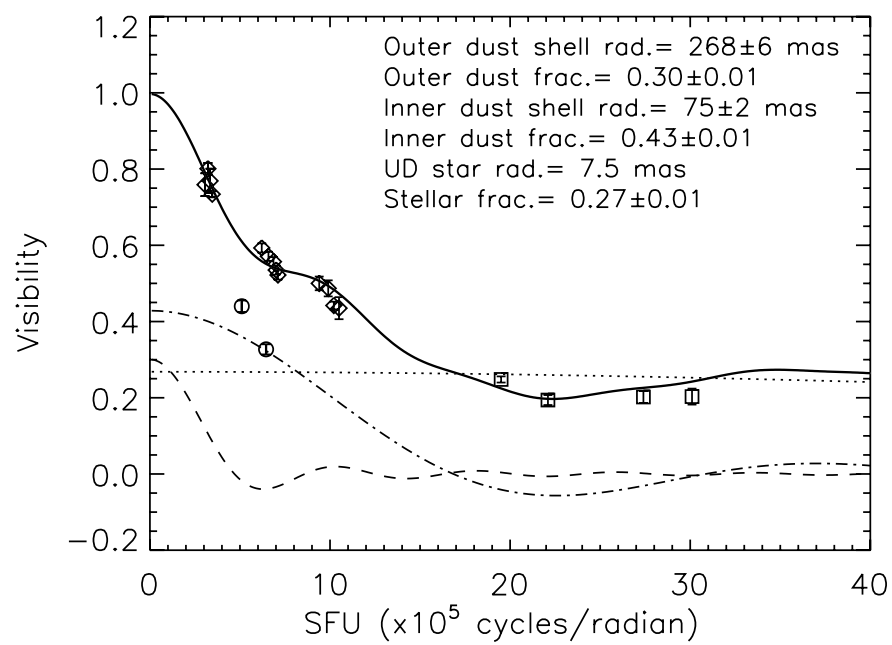

FIG. 1.-Visibility measurements of V Hya at $11.15 \mu \mathrm{m}$ and a theoretical fit involving three uniform disks. The diameters and total flux fractions of the star and dust shells are shown. The horizontal axis is in SFU or "spatial frequency units" of $10^{5}$ cycles $\mathrm{rad}^{-1}$. The diamonds denote visibility data from early 2006, and the squares denote data from late 2006 and early 2007. The solid curve is the model visibility fitted to the data; the dotted, dashed, and dot-dashed curves indicate the stellar, outer, and inner disk components, respectively. The two points shown with circles are from 1997, when the visibility was clearly different from 2006, and they are not included in the fit. component radii and fractions of the total flux, are summarized in Figure 1.

The one-dimensional integrated intensity, shown in Figure 2, is obtained by Fourier transformation of the fitted net visibility function shown in Figure 1. An alternative model, shown in Figure 3, fits the data to visibility curves with a UD representing the star, an inner emission region with a Gaussian one-dimensional intensity distribution, and a UD for the outer dust shell. This model fits the data somewhat better than the three uniform disks model. Figure 4 shows the one-dimensional integrated intensity for the fitted visibilities of Figure 3. For both Figures 3 and 4, the absolute intensity is derived by equating the model total flux density to the absolute flux density at $11.15 \mu \mathrm{m}$ measured by Monnier et al. (1998).

\section{DISCUSSION}

The luminosity phases of the star are also given in Table 1 . Although the measurements of 2006 and 2007 were not made during the same point of the stellar cycle, they have been combined for the modeling. Visibilities at spatial frequencies less than 20 spatial frequency units (SFUs) ( $1 \mathrm{SFU}=1 \times$ $10^{5}$ cycles $\mathrm{rad}^{-1}$ ) were measured in early 2006 , near the stellar maximum. It is possible that visibilities measured later in 2006 and in early 2007, near the stellar minimum and at spatial frequencies greater than $20 \mathrm{SFU}$, are somewhat higher than what

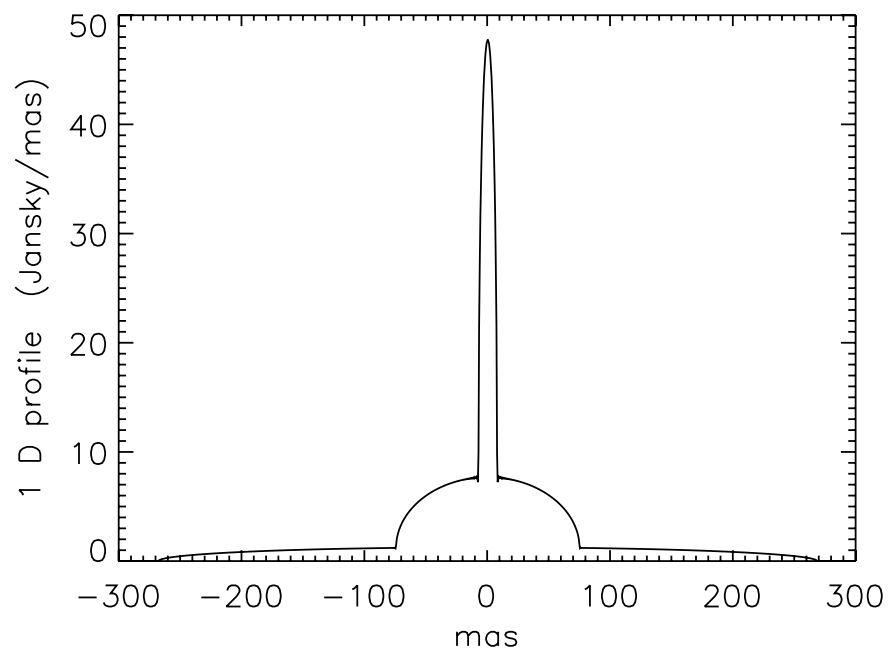

FIG. 2.-The one-dimensional intensity profile of V Hya at $11.15 \mu \mathrm{m}$ obtained from the three-uniform-disk-model visibility curve of Fig. 1. The intensity is plotted as a function of angular radius in units of milliarcseconds. 


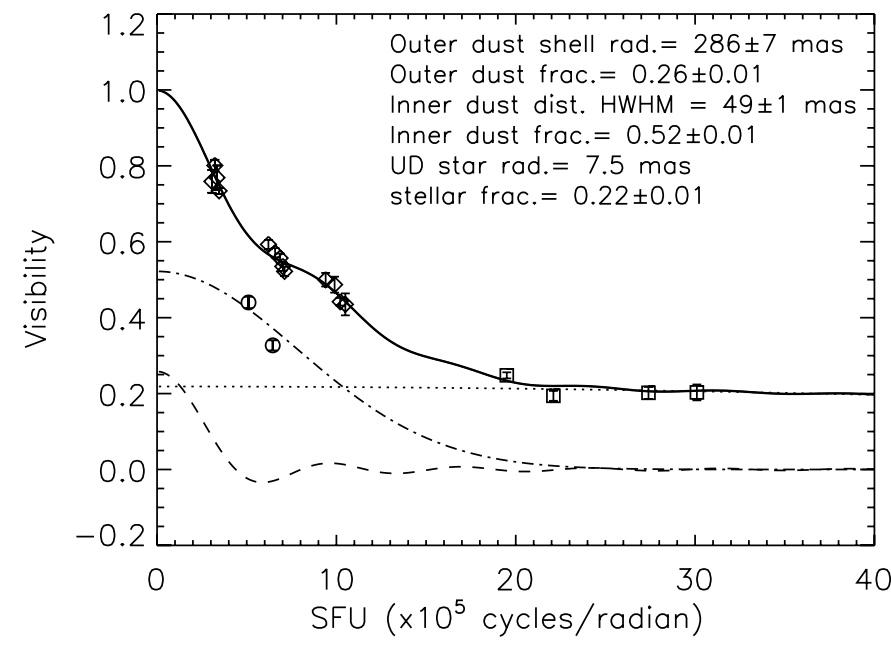

FIG. 3.-Visibility measurements of V Hya at $11.15 \mu \mathrm{m}$ and a theoretical fit involving a stellar UD, a Gaussian intensity distribution, and an outer UD. The sizes and total flux fractions of the star and dust shells are shown. The diamonds denote visibility data from early 2006, and the squares denote data from late 2006 and early 2007. The solid curve is the model visibility fitted to the data; the dotted, dashed, and dot-dashed curves indicate the stellar, outer, and inner components, respectively. The two points shown with circles are from 1997, and they are not included in the fit.

would have been measured near the stellar maximum. This is because Mira variables have their largest size measured in the mid-IR near the stellar luminosity maximum, and their smallest size is near the stellar minimum (e.g., Weiner et al. 2003), and a larger diameter corresponds to a lower visibility at a given spatial frequency within the first lobe of the visibility curve. The change in mid-IR intensity over a luminosity cycle is not particularly large, and we assume that visibilities are consistent and fit them with the same model curve. Since the higher-resolution

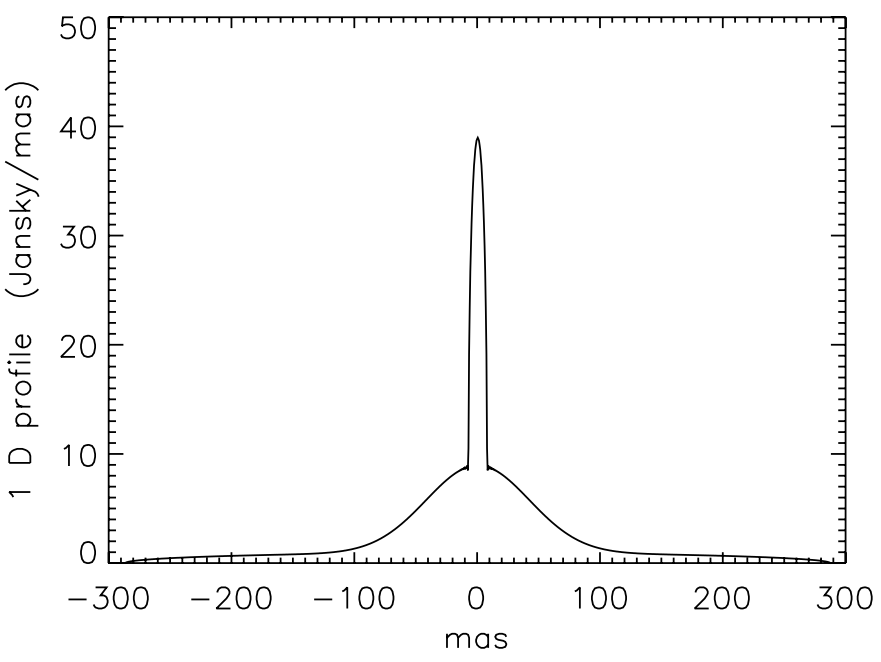

FIG. 4.-The one-dimensional intensity profile of V Hya at $11.15 \mu \mathrm{m} \mathrm{ob}-$ tained from the full visibility curve of Fig. 3. The intensity is plotted as a function of angular radius in units of milliarcseconds. measurements correspond to low visibilities and primarily represent the star, any error in this assumption would not change the fit for the dust distribution appreciably.

Visibility data taken in the late fall of 1997, which are also shown in Figures 1 and 3, are quite different from those of 2006. The 1997 visibility curve has the value unity at zero SFU, by definition, and it falls off sharply to the measured values of 0.44 and 0.33 at 5.1 and $6.45 \mathrm{SFU}$, respectively. This indicates that a dust shell contributes at least a fraction of $\sim 0.7$ to the total flux. A fit of the 1997 points to a model with a Gaussian distribution of dust and a stellar UD of radius 7.5 mas gives a rough estimate of $\sim 0.125^{\prime \prime}$ HWHM (half-width at half-maximum) for the dust shell radius, with a total flux fraction of $\sim 0.75$. It is not surprising that the dust distribution should change over an $8 \mathrm{yr}$ period, and the observed change indicates that the dust is forming episodically. For example, if the star is at a distance of 500 pc (Knapp et al. 1999), a radial velocity of $15 \mathrm{~km} \mathrm{~s}^{-1}$ would lead to a proper motion of $0.5^{\prime \prime}$. Such a velocity is reasonable when compared with the velocities of gas further away from the star, different parts of which have been measured as having deprojected velocities of $16 \mathrm{~km} \mathrm{~s}^{-1}, 40-120 \mathrm{~km} \mathrm{~s}^{-1}$, and $70-185 \mathrm{~km} \mathrm{~s}^{-1}$ (Hirano et al. 2004). Hence, such a change is not unexpected, though the very high velocities measured by Hirano et al. (2004) are thought to be due to winds from an accretion disk and produced some distance from V Hya itself. The low-visibility points of 1997 are far enough away in time and in luminosity phase to be unaffected by the secondary period minimum, which occurred in early 1994 . The secondary minimum is suggested to be due to an eclipsing disk from a companion (Knapp et al. 1999; Sahai et al. 2003).

ISI data from 2006 and 2007 show the presence of two dust shells, and data from 1997 indicate that more dust was present at large scales at this earlier time. Previous near-IR visibilities at $2.2 \mu \mathrm{m}$ from 2000 were fitted with a model consisting of a UD star with radius 7.25 mas and a flux fraction of 0.63 , surrounded by a Gaussian dust distribution of HWHM of 17.5 mas (Millan-Gabet et al. 2003). By comparison, the results of Figures 2 and 4 show an inner dusty region that is considerably larger and has a flux fraction that is also larger. The higher flux fraction and larger mid-IR size are due in part to differences in the dust opacity between the two bands and to the mid-IR sensitivity to lower-temperature material, but changes to the stellar environs have also probably taken place, as indicated by the changes between 1997 and 2007.

While much of the published studies on V Hya can be interpreted within the framework that it is developing bipolar outflows, the present study does not show point-inversion asymmetries that would be indicated by a nonzero closure phase over angular scales of 0.02 to $0.7^{\prime \prime}$. The mid-IR visibility measurements can be modeled using a UD for the star, a Gaussian distribution for an inner emission region, and a UD for a thin outer dust shell. V Hya is an active star emitting much material and it is changing rapidly. Because of these changes, and since the star 
is now near its secondary minimum, additional and continued observations at various wavelengths and resolutions would be particularly interesting.

W. Fitelson, D.D.S. Hale, A.A. Chandler, R.L. Griffith, and $\mathrm{K}$. Tatebe provided excellent technical and observing support for the measurements. We also appreciate the comments of an anonymous referee. This work made use of data from the American Association of Variable Star Observers (AAVSO), the All Sky Automated Survey (ASAS), and the SIMBAD database. We gratefully acknowledge support from the National Science Foundation, The Gordon and Betty Moore Foundation, and the Office of Naval Research.

\section{REFERENCES}

Hale, D. D. S., et al. 2000, ApJ, 537, 998

Hirano, N., et al. 2004, ApJ, 616, L 43

Knapp, G. R., Jorissen, A., \& Young, K. 2003, A\&A, 326, 318

Knapp, G. R., Dobrovolsky, S. I., Ivezić, Z., Young, K., Crosas, M., Mattei, J. A., \& Rupen, M. P. 1999, A\&A, 351, 97

Lagadec, E., Mékarnia, D., de Freitas Pacheco, J. A., \& Dougados, C. 2005, A\&A, 433, 553

Millan-Gabet, R., et al. 2003, Proc. SPIE, 4838, 202
Monnier, J. D., Geballe, T. R., \& Danchi, W. C. 1998, ApJ, 502, 833 Pedretti, E., et al. 2005, BAAS, 37, 1464

Ragland, S., et al. 2006, ApJ, 652, 650

Sahai, R., Morris, M., Knapp, G. R., Young, K., \& Barnbaum, C. 2003, Nature, 426, 261

Sahai, R., Sugerman, B. E. K., \& Hinkle, K. 2009, ApJ, 699, 1015

Weiner, J., Hale, D. D. S., \& Townes, C. H. 2003, ApJ, 588, 1064 